\title{
Isoproterenol-induced Creatine Kinase Leakage in Langendorff-perfused Rat Heart Associated with Significant Myocardial Edema
}

\author{
Keita Odashiro, MD, Shin-ichi Hiramatsu, MD, \\ Toru Maruyama, MD, Yoshikazu KaJI, MD, \\ Shozo Kanaya, ${ }^{1}$ MD, Takehiko Fujino, ${ }^{\prime} \mathrm{MD}$ \\ and Yoshiyuki Nino, MD
}

\begin{abstract}
SUMmary
Since the mechanism of creatine kinase (CK) leakage induced by $\beta$ adrenoceptor activation remains unclear, we studied the effects of incremental application $\left(10^{-9}\right.$ to $\left.10^{4} \mathrm{M}\right)$ of isoproterenol (ISP) on the CK efflux from Langendorff-perfused isolated rat hearts under aerobic conditions. Tissue water content was estimated after the perfusion experiment. ISP-induced dose-dependent CK leakage was noted in a sigmoidal fashion, which showed low temperature-dependency $\left(Q_{0}\right.$ of 2.41$)$, sensitivity to cepharantine $\left(10^{-6} M\right)$ and propranolol $\left(10^{-7}\right.$ to $\left.10^{-6} \mathrm{M}\right)$ without any signs of demand ischemia or oxidant stress. CK liberation was not replicated at all by maneuvers activating cAMPdependent protein kinase (A-kinase). Myocardial edema noted in the control ISP application was ameliorated by exposure to $10^{-6} \mathrm{M}$ propranolol or cepharantine (i.e., significant fall in tissue water content; $p<0.05$ ). Histological study revealed nonspecific myocardial fiber swelling and separation without any myocyte necrosis for all the perfusion groups. These results suggest that ISP-induced CK leakage in this model is not mediated by $\beta$-adrenoceptor stimulation, subsequent A-kinase activation or related demand ischemia, but is attributed most to the direct effects of ISP augmenting sarcolemmal CK and water permeability. (Jpn Heart J 1998; 39: 513-525)
\end{abstract}

Key words: $\beta$-adrenoceptor, cAMP-dependent protein kinase, Creatine kinase, Myocardial edema, Sarcolemma

\footnotetext{
YREATINE kinase (CK) is an established marker of the onset of irreversible myocardial injury. This enzyme is utilized in the assessment of the myocardial infarct size quantitation and of the onset of coronary reperfusion in both basic $^{1,2)}$ and clinical fields. ${ }^{3,4)}$ On the other hand, pharmacological modulation of myocardial CK leakage has been observed. Stimulation of $\beta$-adrenoceptor re-

From the First Department of Internal Medicine, Faculty of Medicine, Kyushu University, Fukuoka, Japan, and 'The Institute of Health Science, Kyushu University, Kasuga, Japan.

Address for correspondence: Toru Maruyama, MD, The First Department of Internal Medicine, Faculty of Medicine, Kyushu University, Maidashi 3-1-1, Higashi-ku, Fukuoka 812-8582, Japan.

Received for publication February 18, 1998

Revised and accepted April 22, 1998.
} 
veals versatile and profound cardiovascular influences, including myocardial CK liberation. ${ }^{5}$ ) However, the exact mechanism of myocardial GK release caused by $\beta$-adrenoceptor activation is poorly understood. It is reasonable to assume that resolution of this question will contribute greatly to a better understanding of socalled catecholamine cardiotoxicity, ${ }^{6}$ and the prevention of this cardiotoxicity will also contribute to the establishment of a clinical cardioprotective strategy. Therefore, in the present study, we have investigated i) the effects of $\beta$ adrenoceptor stimulation afforded by isoproterenol (ISP) application to the Langendorff-perfused rat heart model on CK leakage and some other associated phenomena and ii) the effects of $\beta$-adrenoceptor blockade caused by propranolol, which was expected to exert cardioprotective actions against these undesired phenomena.

\section{Materials and Methods}

Heart preparation: The study was performed according to the Guiding Principles for the Care and Use of Animals approved by the Council of the Japanese Physiological Society. Adult male Wistar rats (Kyudo Co Ltd., Yoshitomi, Japan) were given a commercial diet (Japan Clea, Tokyo, Japan) and tap water ad libitum in the Kyushu University Animal Center. Rats weighing 250 to $350 \mathrm{~g}$ were anesthetized with intraperitoneal sodium pentobarbital $(50 \mathrm{mg} / \mathrm{kg}$; Abbott Laboratory, North Chicago, IL) and the beating heart was removed immediately after thoracotomy under artificial respiration (KN-55, Natsume, Tokyo). Blood was rinsed out in ice-cold Krebs-Henseleit $(\mathrm{K}-\mathrm{H})$ solution and the aorta was cannulated for the modified Langendorff perfusion. The composition of the K-H solution was $(\mathrm{mM}) \mathrm{NaCl} 118.5, \mathrm{KCl} 4.7, \mathrm{NaHCO}_{3} 25.0, \mathrm{CaCl}_{2}$ 1.4, $\mathrm{MgSO}_{4} 1.2$, $\mathrm{KH}_{2} \mathrm{PO}_{4} 1.2$ and glucose 5.5. The $\mathrm{K}-\mathrm{H}$ solution was gassed with $95 \% \mathrm{O}_{2}-5 \%$ $\mathrm{CO}_{2}$ to maintain a $\mathrm{pH}$ of 7.4 and warmed at $36^{\circ} \mathrm{C}$. The partial oxygen pressure $\left(\mathrm{PO}_{2}\right)$ estimated by a blood gas analyzer (ABL 620; Radiometer, Copenhagen, Denmark) was approximately $300 \mathrm{mmHg}$. Coronary flow rate was regulated by a peristaltic perfusion pump (model 7553-20, Cole-Parmer, Chicago, IL) at 14 to $16 \mathrm{ml} / \mathrm{min}$. The flow rate was not changed during an experiment. Consequently, measured perfusion pressure was distributed in the range of 50 to $60 \mathrm{mmHg}$.

The left atrium was removed and the left ventricular developed pressure (LVDP) produced by isovolumetric contractions was measured with a latex balloon inserted into the left ventricle through the mitral orifice and connected to the digital manometer (Tsukasa-Sokken, Tokyo). Left ventricular end-diastolic pressure was adjusted to about $5 \mathrm{mmHg}$. ECG was monitored using a 1-channel ECG amplifier (Nihon Kohden Corporation, Tokyo) by setting a pair of clip electrodes on the epicardium. An indifference electrode was placed on the 
cannulated ascending aorta. These signals were transferred to a pen-writing recorder (Nihon Kohden) and to a casette tape recorder (TEAC, Tokyo) for subsequent analysis. Coronary effluent sampling was started $30 \mathrm{~min}$ after equilibration and then continued sequentially. Hearts with visible epicardial coronary thrombi or air emboli and those with LVDP less than $50 \mathrm{mmHg}$ were excluded from the analyses. Various ventricular or supraventricular arrhythmias were defined according to the Lambeth Convention. ${ }^{7}$ Preparations showing these arrhythmias persistently before or during the treatment with various agents were also excluded. CK activity in effluent was evaluated immediately after the perfusion experiment by a spectrophotometric kinetic procedure first described by Rosalki ${ }^{83}$ using a commercially available CK assay kit (Wako Chemical Co. Ltd., Tokyo). Effluent lactate concentration was evaluated by the method of Hohorst. ${ }^{9 / 4}$ Sarcolemmal lipid peroxidation was estimated by the formation of thiobarbituric acid-reactive malonedialdehyde (MDA). The method for measuring effluent MDA concentration has been described elsewhere. ${ }^{(0)}$

Perfusion experiment protocol: The first series of experiments $(n=10)$ was designed to establish the dose-response relationship between CK release and concentration of ISP (Sigma Co. Ltd., St Louis, MO), a non-selective $\beta$-agonist. ISP was applied continuously at the range of $10^{-9}$ to $10^{-4} \mathrm{M}$ in an incremental manner. During the application of ISP at any given dose, the preparation was equilibrated $30 \mathrm{~min}$ and then effluent sampling started. The ISP concentration of the initial application was not necessarily $10^{-9} \mathrm{M}$ to limit the total perfusion period to less than 3.5 hours. Some preparations $(n=3)$ were devoted to investigate the temperature dependence of the established dose-response relationship. Incremental ISP application was repeated with two different temperature perfusates $\left(26\right.$ and $\left.30^{\circ} \mathrm{C}\right)$. The second series of experiments was designed to investigate the effects of two different doses of propranolol $\left(10^{-7}\right.$ and $10^{-6} \mathrm{M}, n=6$ for each) on the ISP-induced, dose-dependent myocardial CK leakage. Pretreatment with propranolol (either $10^{-7}$ or $10^{-6} \mathrm{M}$ ) for $30 \mathrm{~min}$ was performed prior to the application of K-H solution containing ISP. Thereafter, incremental ISP application was repeated. Experiments exploring the effects of pretreatment with cepharantine $\left(10^{-6} \mathrm{M}\right)$ on ISP-induced $\mathrm{CK}$ release were conducted in the same way $(n=5)$. The third series of experiments $(n=15)$ was designed to investigate the effects of various drugs on myocardial CK liberation. Forskoline (FSK; Sigma), isobutylmethyl-xanthine (IBMX; Sigma), dibutyryl cyclic AMP (dbcAMP; Sigma) and digoxin (Chugai Pharmaceutical Co. Ltd., Tokyo) were applied independently in this manner. The concentration ranges of these agents were determined arbitrarily, so that the inotropic response obtained with these ligands were roughly equivalent to that observed by ISP. The concentration range was $10^{-8}$ to $10^{-4} \mathrm{M}$ for $\mathrm{FSK}, 10^{-7}$ to $10^{-4} \mathrm{M}$ for IBMX, $10^{-6}$ to $10^{-4} \mathrm{M}$ for 
db-cAMP and $10^{-7}$ to $10^{-4} \mathrm{M}$ for digoxin. The duration in the perfusion of all these agents was adjusted to 3 to 3.5 hours. In the last series of experiments $(n=4)$, whole hearts perfused without any drugs were rapidly paced at high right atrium using a pair of stimulating clip electrodes at a fixed high rate equivalent to that observed by the maximum dose $\left(10^{-4} \mathrm{M}\right)$ of ISP administered (the maximum pacing rate of $400 \mathrm{bpm}$ ) for 3.5 hours. Another four hearts were perfused without any drugs or rapid pacing as a control for the aforementioned long-term (3 to 3.5 hours) perfusion.

Tissue water content estimation: At the end of each experiment half of the preparations were used to estimate tissue water content. Whole hearts were weighed immediately after the perfusion experiment and two days after oven drying. Relative myocardial water content (WC\%) was estimated by the equation,

$$
\mathrm{WC} \%=(\mathrm{WW}-\mathrm{DW}) / \mathrm{WW}
$$

where WWV and DW were wet weight and dry weight, respectively.

Histological study: The other half of the preparations were perfused at the end with K-H solution containing trypan blue $(10 \mathrm{mg} / \mathrm{d} l)$ and then washed out with control K-H solution. After staining with trypan blue, the preparations were perfused with Karnovsky's fixative, removed from the perfusion apparatus, stored in this fixative for 48 hours, and then embedded in paraffin wax. Short-axis sections 4 to 5 microns thick were prepared and stained with hematoxylin and eosin. Histological examination was conducted by a pathologist who was unaware of the experimental conditions.

Data analysis: Nonlinear regression of myocardial CK leakage as a function of ISP concentration was determined using Hill's equation,

$$
\mathrm{CK}=\mathrm{CK}_{\max } \cdot \mathbf{I}^{\mathrm{n}} /\left(\mathrm{I}^{\mathrm{n}}+\mathrm{K}_{\mathrm{d}^{\mathrm{n}}}\right)+\mathrm{C}
$$

where $\mathrm{CK}_{\max }$ was the assumed maximum effluent $\mathrm{CK}$ activity, $\mathrm{C}$ the basal effluent CK activity, I the ISP concentration applied, $\mathrm{K}_{\mathrm{d}}$ the affinity constant and $n$ Hill's coefficient. All data are presented as mean \pm SD. Statistical analysis for comparing WC\% was performed by two-sided nonpaired Student's $t$-test and $p<0.05$ was considered as statistically significant. Non-linear fitting of the doseresponse in the ligand-induced CK efflux and the associated inotropic or chronotropic response to Hill's equation was conducted by the least-square method.

\section{Results}

Perfusion study: In the first protocol, ISP revealed dose-dependent positive 
inotropic and chronotropic actions, yielding sigmoidal curves for heart rate and relative LVDP as a function of ISP concentration. When the dose-response curves were fitted to Hill's equation, $\mathrm{K}_{\mathrm{d}}$ values of $3.2 \times 10^{-10} \mathrm{M}$ for heart rate and $9.7 \times 10^{-9} \mathrm{M}$ for LVDP were obtained $(n=10)$. In the second protocol, pretreatment with propranolol (up to $10^{-6} \mathrm{M}$ ) prior to the administration of ISP resulted in competitive reduction in the inotropic and chronotropic responses $(n=6) . \mathrm{K}_{\mathrm{d}}$ shifted to the higher concentration for both heart rate $\left(0.8 \times 10^{-7} \mathrm{M}\right)$ and LVDP $\left(2.9 \times 10^{-7} \mathrm{M}\right)$. In the third protocol, a positive inotropic response was induced by treatment with digoxin $\left(10^{-7}\right.$ to $\left.10^{-4} \mathrm{M}, n=4\right)$ or mancuvers established to activate the intracellular A-kinase such as exposure to FSK $\left(10^{-7}\right.$ to $\left.10^{-4} \mathrm{M}, n=4\right)$, a non- $\beta$-mediated adenylcyclase activator, $\operatorname{IBMX}\left(10^{-6}\right.$ to $\left.10^{-4} \mathrm{M}, n=3\right)$, a nonspecific phosphodiesterase inhibitor, and db-cAMP $\left(10^{-6}\right.$ to $\left.10^{-4} \mathrm{M}, n=4\right)$, a membrane-permeable cAMP analogue. The efficacy of positive inotropism evaluated by the respective $K_{d}$ values for these agents was in the order of ISP $>$ FSK > digoxin > IBMX > db-cAMP. On the other hand, the potencies (i.e., the maximum \% increase in LVDP) were in the order of ISP $=$ FSK $>$ digoxin $\geq \mathrm{db}$-cAMP $>$ IBMX (Figure 1). Except for digoxin, these agents presented positive chronotropism. Similarly, the efficacy of positive chronotropism based on $\mathrm{K}_{\mathrm{d}}$ for these ligands was in the order of

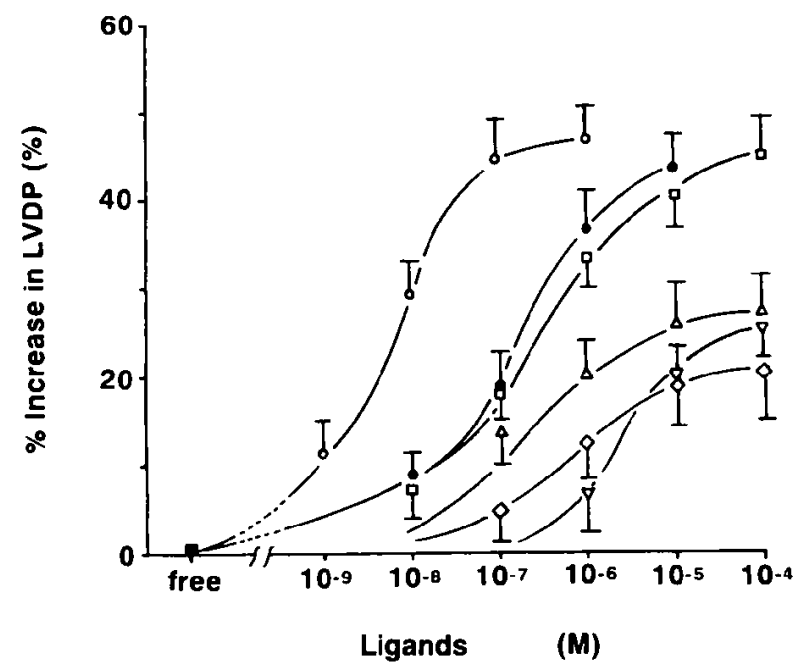

Figure 1. The dose-response relationship of positive inotropism observed by various ligands such as isoproterenol $\langle$ ISP; $O, n=10\rangle$, forskoline (FSK; $\square, n=4)$, digoxin $(\triangle$, $n=4)$, dibutyryl cyclic AMP (db-cAMP; $\nabla, n=4)$ and isobutylmethylxanthine (IBMA; $\vartheta, n=3)$. The dose response curve obtained by pretreatment with propranolol $\left(10^{-7}\right.$ M) and subsequent ISP is also shown $(0, n=6)$. All of the sigmoidal curves were fitted by Hill's equation. Symbols and bars indicate mean \pm SD. 


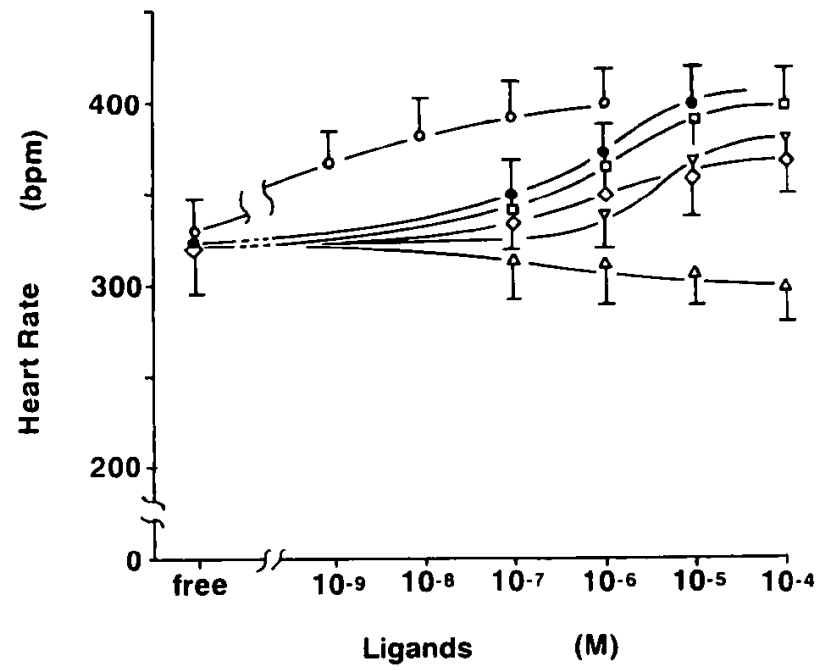

Figure 2. The dose-response relationship of positive and negative chronotropism observed by various ligands listed in Figure 1. The symbols are the same as in Figure 1. Note the modest negative chronotropism obtained by treatment with digoxin. The sigmoidal curve of heart rate as a function of ISP concentration was interrupted because of the small $K_{r}$ value. All of the sigmoidal curves were determined using Hill's equation. Symbols and bars indicate mean $\pm \mathrm{SD}$

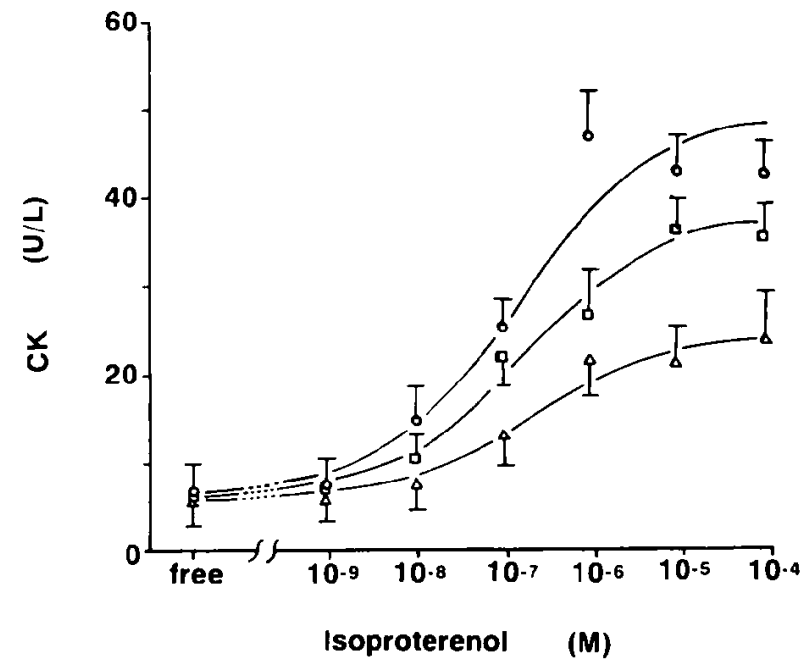

Figure 3. Temperature dependency of the ISP-induced myocardial leakage of creatine kinase $(\mathrm{CK})$. The dose-response curves were obtained under perfusion at three different temperatures $\left(\bigcirc\right.$ at $36^{\circ} \mathrm{C}, \square$ at $30^{\circ} \mathrm{C}$ and $\triangle$ at $26^{\circ} \mathrm{C}, n=3$ ). These curves were determined using Hill's equation. Temperature coefficient was estimated as 2.41. Symbols and bars indicate mean \pm SD. 
ISP $\gg$ FSK > IBMX > db-cAMP, whereas the order of the potency (i.e., the maximum increase in the average heart rate) was $I S P=F S K>d b-$ cAMP > IBMX (Figure 2).

Enzymatic study: In the first series of experiments applying incremental ISP at $36^{\circ} \mathrm{C}(n=10)$, myocardial CK leakage was augmented in a dose-dependent manner. This tendency afforded a nonlinear regression fitted to Hill's equation associated with $\mathrm{CK}_{\max }=45.7 \mathrm{IU} / \mathrm{L}$ and $\mathrm{K}_{\mathrm{d}}=1.77 \times 10^{-7} \mathrm{M}$. Perfusion at $30^{\circ} \mathrm{C}$ yielded a sigmoidal dose-response relationship between $\mathrm{CK}$ and ISP characterized by $\mathrm{CK}_{\max }=30.4 \mathrm{IU} / \mathrm{L}$ and $\mathrm{K}_{\mathrm{d}}=1.69 \times 10^{-7} \mathrm{M}$. Further lowering the perfusate temperature to $26^{\circ} \mathrm{C}$ yielded a sigmoidal dose-response curve with a $\mathrm{CK}_{\max }=19.2$ $\mathrm{IU} / \mathrm{L}$ and $\mathrm{K}_{\mathrm{d}}=1.80 \times 10^{-7} \mathrm{M}(n=3)$. These findings revealed temperature-dependent $\mathrm{CK}_{\max }$ alteration, identical $\mathrm{K}_{\mathrm{d}}\left(1.77 \times 10^{-7} \mathrm{M}\right), n(0.5 \mathrm{l})$ and $\mathrm{C}(7.4 \mathrm{IU} / \mathrm{L})$, and a temperature coefficient of 2.41 in the ISP-induced CK efflux (Figure 3).

Propranolol exerted concentration-dependent characteristic effects on ISPinduced myocardial CK liberation. The control dose-response relationship of the ISP-induced $\mathrm{CK}$ leakage was suppressed $\left(\mathrm{CK}_{\max }=25.9 \mathrm{IU} / \mathrm{L}\right)$ and shifted to the left along the abscissa $\left(\mathrm{K}_{\mathrm{d}}=2.48 \times 10^{-8} \mathrm{M}\right)$ by pretreatment with $10^{-7} \mathrm{M}$ propranolol $(n=6)$. On the other hand, application of $10^{-6} \mathrm{M}$ propranolol resulted in a sigmoidal relationship with CK leakage and ISP application was

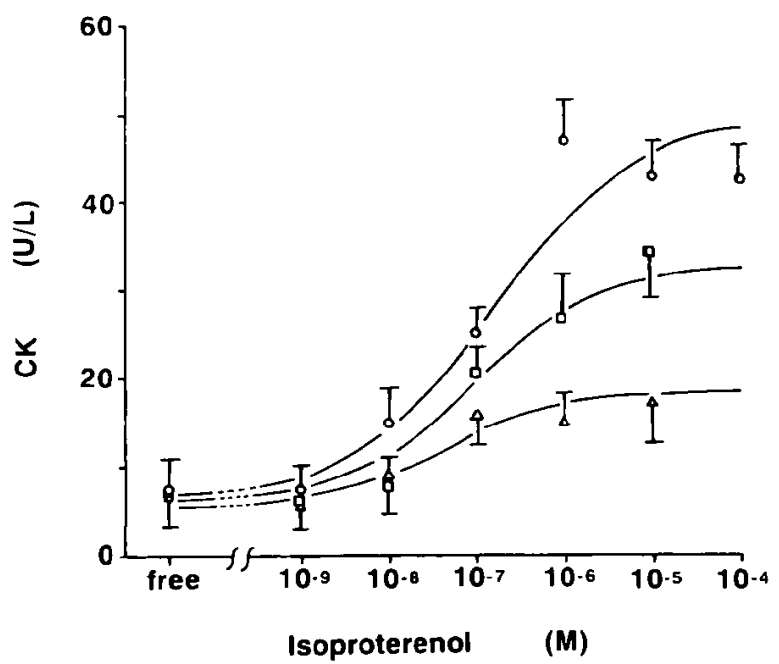

Figure 4. The effects of pretreatment with two different doses of propranolol $\left(10^{-7}\right.$ and $10^{-6} \mathrm{M}$ ) on ISP-induced myocardial CK efflux. Note the dose-dependent fall in $\mathrm{CK}_{\max }$ obtained by the control ISP application $(\mathrm{O}, n=10)$ by the pretreatment with two different doses of propranolol $\left(10^{-7} \mathrm{M} ; \square, n=6\right.$ and $\left.10^{-6} \mathrm{M}: \Delta, n=6\right)$. All of the sigmoidal curves were determined using Hill's equation. Symbols and bars indicate mean $\pm \mathrm{SD}$. 


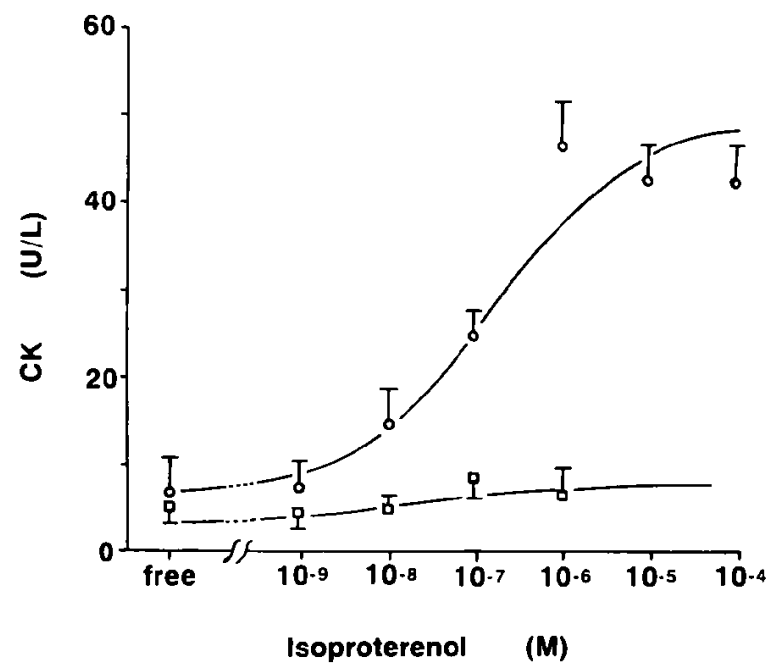

Figure 5. The effects of cepharantine on the ISP-induced myocardial CK leakage. Note that control CK liberation $(\mathrm{O}, n=10)$ was completely dissipated by pretreatment with cepharantine $(\square, n=5$ ). Sigmoidal curves were determined using Hill's equation. Symbols and bars indicate mean $\pm \mathrm{SD}$.

Table. Comparison of Wet Weight (WW), Dry Weight (DW) and Relative Water Content (WC\%) of the Perfused Whole Hearts Exposed to Various Maneuvers

\begin{tabular}{lllll}
\hline & & WW $(\mathrm{g})$ & $\mathrm{DW}(\mathrm{g})$ & WC\% (\%) \\
\hline control & & $1.59 \pm 0.21$ & $0.60 \pm 0.17$ & $62.3 \pm 3.0$ \\
rapid pacing & $(\sim 400 \mathrm{bpm})$ & $1.56 \pm 0.24$ & $0.59 \pm 0.21$ & $62.1 \pm 2.9$ \\
digoxin & $\left(\sim 10^{-4} \mathrm{M}\right)$ & $1.70 \pm 0.18$ & $0.60 \pm 0.19$ & $64.8 \pm 2.4$ \\
db-cAMP & $\left(\sim 10^{-4} \mathrm{M}\right)$ & $1.64 \pm 0.20$ & $0.59 \pm 0.14$ & $63.9 \pm 2.6$ \\
IBMX & $\left(\sim 10^{-4} \mathrm{M}\right)$ & $1.61 \pm 0.17$ & $0.56 \pm 0.13$ & $65.1 \pm 2.0$ \\
FSK & $\left(\sim 10^{-4} \mathrm{M}\right)$ & $1.66 \pm 0.19$ & $0.59 \pm 0.17$ & $64.3 \pm 2.3$ \\
cepharantine & $\left(\sim 10^{-6} \mathrm{M}\right)+\mathrm{ISP}$ & $1.77 \pm 0.20$ & $0.58 \pm 0.14$ & $66.9 \pm 2.8$ \\
propranolol & $\left(10^{-6} \mathrm{M}\right)+\mathrm{ISP}$ & $1.89 \pm 0.16^{*}$ & $0.54 \pm 0.15$ & $71.3 \pm 2.1$ \\
propranolol & $\left(10^{-7} \mathrm{M}\right)+\mathrm{ISP}$ & $2.03 \pm 0.19^{*}$ & $0.53 \pm 0.16$ & $74.2 \pm 3.0^{*}$ \\
ISP & $\left(\sim 10^{-4} \mathrm{M}\right)$ & $2.12 \pm 0.21^{*}$ & $0.58 \pm 0.24$ & $75.7 \pm 2.4^{*}$ \\
\hline
\end{tabular}

db-cAMP = dibutyryl cyclic AMP; FSK = forskoline; IBMX = isobutylmethylxanthine; ISP = isoproterenol; $* p<0.05$ compared to the control.

further suppressed $\left(\mathrm{CK}_{\max }=15.0 \mathrm{IU} / \mathrm{L}\right)$ with a $\mathrm{K}_{\mathrm{d}}$ value of $2.40 \times 10^{-8} \mathrm{M}(n=6)$. All of these regression analyses had identical $n=0.50$ and $\mathrm{C}=7.6 \mathrm{IU} / \mathrm{L}$ (Figure 4).

Cepharantine $\left(10^{-6} \mathrm{M}\right)$, a potent sarcolemmal stabilizer, caused profound suppression of myocardial CK leakage as a function of ISP concentration $(n=5)$, yielding the regression results $\mathrm{CK}_{\max }=8.6 \mathrm{IU} / \mathrm{L}$ and $\mathrm{K}_{\mathrm{d}}=1.34 \times 10^{-8} \mathrm{M}$ (Figure 5). Effluent lactate and MDA were not detected persistently throughout the experiments when ISP was applied alone or in combination with propranolol or 
cepharantine. The application of FSK, IBMX, db-cAMP or digoxin caused no significant incremental CK leakage. During all these procedures, effluent CK activity was $10 \mathrm{IU} / \mathrm{L}$ or less and effluent lactate and MDA were undetectable. Rapid right atrial pacing at a fixed high rate (up to $400 \mathrm{bpm}$ ) and a duration of 3.5 hours also resulted in no detectable lactate release and no increase in efflux of $\mathrm{CK}>10 \mathrm{IU} / \mathrm{L}$ under aerobic conditions $\left(\mathrm{PO}_{2} \approx 300 \mathrm{mmHg}, n=4\right)$.

Tissue water content study: The mean wet and dry weight of the heart as well as the relative tissue water content (WC\%) under the various perfusion protocols are summarized in the Table. WC\% of heart perfused without any drugs in the absence of high right atrial rapid pacing was considered to be the standard. WC\% of hearts perfused with no drugs under rapid pacing showed no statistical difference. WC\% in the case of application of digoxin, db-cAMP, IBMX or FSK was also within standard levels. WC\% values in the ISP application (up to $10^{-4} \mathrm{M}$ ) and the pretreatment with cepharantine or $10^{-6} \mathrm{M}$ propranolol were not significantly different from standard WC\%. On the other hand, WC\% in the control ISP application (up to $10^{-4} \mathrm{M}$ ) was significantly $(p<0.05)$ greater than the standard WC\%. The same was true in the case of ISP application (up to $10^{-4} \mathrm{M}$ ) and the pretreatment with $10^{-7} \mathrm{M}$ propranolol. This implies a dose-dependent inhibitory effect of propranolol on the ISP-induced gain in myocardial water content.

Histological study: In all preparations examined histologically, slight swelling and the separation of individual myofibers were observed, which was independent of the difference in the perfusion protocols. Although quantitation of these histological findings was not undertaken, the extent of these findings was not qualitatively different between control ISP application and the other experimental protocols. Myocyte necrosis characterized by the strong nuclear staining and slight cytosolic staining with trypan blue was not evident. A small amount of trypan blue deposit was observed in the intravascular space and perivascular interstitium, which was considered to be a result of incomplete washout immediately after the trypan blue staining.

\section{Discussion}

The first major finding of the present study is that incremental ISP application resulted in the myocardial CK leakage and a gain in the tissue water content which indirectly suggested myocardial edema. The second finding is that ISPinduced CK efflux and edema formation were not replicated by A-kinase activation caused by FSK, IBMX or db-cAMP, in spite of the positive inotropic and chronotropic responses observed by applying these agents. The third is the pretreatment with propranolol prevented both myocardial CK liberation and edema 
in a dose-dependent manner.

Myocardial CK release has been shown to be regulated by several neurohumoral factors such as insulin, ${ }^{11)}$ digitalis compounds, ${ }^{12)} \beta$-blocking agents and vagal activity. ${ }^{13)}$ In the present study, it is evident that ISP induced dose-dependent CK efflux and concomitant myocardial edema. Many conflicting cardiotonic, cardiotrophic and cardiotoxic evidence with respect to ISP in in vivo rat studies has been reported. $\left.{ }^{6}\right)$ Rona et al. were first to describe the infarct-like myocardial necrosis induced by a single subcutaneous injection of ISP (up to 680 $\mathrm{mg} / \mathrm{kg})^{1+1,15)}$ Fleckenstein et al. explained the ISP cardiotoxicity in terms of ISPinduced massive $\mathrm{Ca}^{2+}$ entry leading to intracellular $\mathrm{Ca}^{2+}$ overload followed by mitochondrial dysfunction and high energy phosphate breakdown. ${ }^{16)}$ These sequential metabolic deterioration events induce structural damage in the cytoskeleton and sarcolemma partly by activating $\mathrm{Ca}^{2+}$-dependent phospholipase. On the other hand, daily subcutaneous injections ( 50 to $5000 \mu \mathrm{g} / \mathrm{kg}$ ) or intravenous infusion (1 to $22 \mathrm{mg} / \mathrm{kg} /$ day) for up to 10 days caused myocardial fibrosis secondary to the diffuse myocyte loss ${ }^{17}$ and daily subcutaneous injections of ISP ( $2500 \mu \mathrm{g} / \mathrm{kg}$ ) for a week has been reported to evoke myocardial hypertrophy ${ }^{18)}$ These discrepancies are largely dependent on the different experimental designs, such as the dose, duration and form of ISP administered.

Contrary to these in vivo chronic studies, there is less data on the acute effects of ISP on the isolated rat heart. It is unlikely in the histological examination that the myocardial CK release observed in the present study was due to the ISP-induced myocyte necrosis. It is also unlikely that ISP caused myocardial demand ischemia due to an increase in oxygen demand since effluent lactate was not detected in any hearts perfused under completely aerobic conditions $\left(\mathrm{PO}_{2} \approx 300 \mathrm{mmHg}\right)$. The fact that ISP is easily autooxidized and propranolol is a potent antioxidant agent ${ }^{19)}$ suggests that the undesirable effects of ISP are mediated by the sarcolemmal oxidant stress. However, the lack of any detectable effluent MDA in all of the perfusion protocols leads us to the conclusion that oxidant stress and the resultant sarcolemmal peroxidation were not the main features of ISP-induced CK liberation. Therefore, A-kinase activation secondary to the $\beta$-adrenoceptor stimulation is thought to be responsible for the ISP-induced myocardial CK release. A-kinase is assumed to have several phosphorylating targets on membrane proteins, which influences sarcolemmal permeability. Therefore, we applied agents such as FSK, which activates adenylcyclase directly, IBMX which inhibits phosphodiesterase and hence preserves cAMP, and db-cAMP, which is a membrane-permeable cAMP analogue. Circumferential evidence of A-kinase activation by these procedures was obtained from the positive inotropic and chronotropic response (Figures 1 and 2). However, unexpectedly, none of these procedures had a substantial effect on myocardial CK leakage 
in our perfusion study. The relatively low temperature coefficient for ISP-induced CK efflux $\left(Q_{0}=2.41\right)$ also implies that bioactive processes such as cAMP production are unlikely causes of ISP-induced CK liberation. Bernauer reported on digoxin-induced myocardial $\mathrm{CK}$ release using perfused guinea pig whole hearts, ${ }^{12)}$ and indicated that the myocardial CK leakage was attributable to the positive inotropism obtained with digoxin. Therefore, the effects of positive inotropism and chronotropism not mediated by $\beta$-adrenoceptor stimulation on myocardial CK leakage were explored. Positive inotropic and chronotropic action equivalent to ISP application was replicated by digoxin treatment and rapid high atrial pacing, respectively. However, these maneuvers had no substantial influence on the myocardial CK liberation, indicating the positive inotropism or chronotropism to an extent observed in our model could not release CK. The discrepant results with respect to the digoxin-induced CK liberation between our study and the report by Bernauer is presumably attributable to species differences for digitalis sensitivity between rats and guinea pigs. ${ }^{20,21)}$

Based on these negative experimental results, a direct sarcolemmal action of ISP was assumed to be responsible for myocardial CK leakage. The dose-dependent inhibitory effects of propranolol on this leakage are noteworthy. Pharmacological analysis provided the interpretation that $\beta$-adrenoceptor blockade caused by $10^{-7} \mathrm{M}$ propranolol evoked competitive inhibition of the ISP-induced positive inotropism and chronotropism (shift in $\mathrm{K}_{d}$ to the right in Figures 1 \& 2) and noncompetitive suppression of ISP-induced CK efflux (fall in $\mathrm{CK}_{\max }$ in Figure 4) simultaneously. A higher dose of propranolol $\left(10^{-6} \mathrm{M}\right)$ accentuated this noncompetitive antagonism (further fall in $\mathbf{C K}_{\max }$ ). Based on the pharmacological analysis of the effects of propranolol and the replication of ISP with the aforementioned procedures, the sharp contrast between the physiological and enzymatic results was dependent on the A-kinase-mediated signaling pathway. Propranolol has been reported to exert various pharmacological actions not mediated by $\beta$ adrenoceptor blockade. These include antioxidant effects ${ }^{19}$ and sarcolemmal stabilizing effects such as phospholipid preservation. ${ }^{22)} \mathrm{A}$ non- $\beta$-adrenoceptor mediated protective action of high doses of propranolol $(20 \mu \mathrm{M})$ was reported in perfused rat hearts subjected to hypoxia/reoxygenation. ${ }^{23)}$ Okumura et al. demonstrated the attenuation of CK leakage in in vivo rats administered ISP $(40 \mathrm{mg}$ / $\mathrm{kg}$ ) by pretreatment with chlorpromazine, a nonspecific phospholipase inhibitor. They concluded that sarcolemmal phospholipid preservation was important for maintaining myocardial sarcolemmal integrity. ${ }^{24)}$ Our results clarifying the cardioprotective actions of propranolol, perhaps via sarcolemmal stabilization not mediated by $\beta$-adrenoceptor blockade, resemble those for chlorpromazine. In the various perfusion experiments in this study, the extent of CK leakage was in good accordance with that of edema formation, indicating that the myocardial edema 
noted in this study was a result of water influx counteracting CK efflux and that the novel antiedema effects observed only with the high dose $\left(10^{-6} \mathrm{M}\right)$ of propranolol was ascribable to the non- $\beta$-adrenoceptor mediated sarcolemmal protection.

This study has several limitations. Although MDA production was estimated, ascorbic acid or sodium metabisulfite should have been added to the perfusate to prevent the autooxidation of ISP. ${ }^{17}$ Myocardial MDA formation should have been estimated using the extracted cardiac tissue, and the effluent MDA assay is not perfect. Myocardial ischemia should be precisely evaluated by quantifying myocardial high energy phosphate compounds and oxygen consumption estimatcd by comparing $\mathrm{PO}_{2}$ in the perfusate prior to and immediately after coronary circulation. Moreover, light microscopic examination of the myocyte necrosis is incomplete. Electron microscopy may reveal subcellular damage in myocytes which appeared normal (i.e., negative in trypan blue) under light microscopy.

In conclusion, ISP-induced myocardial CK leakage as well as edema formation were not replicated by the A-kinase activation caused by FSK, IBMX or dbcAMP and the dose-dependent protective effects of propranolol on these phenomena were observed, indicating ISP and propranolol have non- $\beta$ adrenoceptor-mediated toxic and protective effects.

\section{Acknowledgements}

The authors wish to thank Dr. Nobuhito Yanagi, MD, for his assistance with the pathology examinations and Ms. Yayoi Kitagawa-Yanagi for her excellent secretarial assistance.

\section{References}

1. Shell WE, Kjekshus JK, Sobel BE. Quantitative assessment of the extent of myocardial infarction in the conscious dog by means of analysis of serial changes in serum creatine phosphokinase activity. $J$ Clin Invest $1971 ; 50: 2614-25$.

2. Vatner SF, Baig H, Manders WT, Maroko PR. Effects of coronary artery reperfusion on myocardial infarct size calculated from creatine kinase. J Clin Invest 1978; 61: 1048-56.

3. Adams JE, Abendschein DR, Jaffe AS. Biochemical markers of myocardial injury: is MB creatine kinase the choice for the 1990s? Circulation 1993; 88: 750-63.

4. Lee TH, Goldman L. Serum enzyme assays in the diagnosis of acute myocardial infarction. Ann Intern Med 1986; 105: 221-33.

5. de Leiris J, Hearse DJ. Myocardial enzyme leakage as an indicator of cellular injury: principles and application. In: Dhalla NS, editor. Methods in Studying Cardiac Membranes, Vol. 1. Florida: CRC Press, 1984: 253-77.

6. Rona G. Catecholamine cardiotoxicity. J Mol Cell Cardiol 1985; 17: 291-306.

7. The Lambeth conventions. Guidelines for the study of arrhythmias in ischaemia, infarction, and reperfusion. Cardiovasc Res 1988; 22: 447-55. 
8. Rosalki SB. An improved procedure for serum creatine phosphokinase determination. J Lab Clin Med 1967; 69: 696-705.

9. Hohorst HJ. $\mathrm{L}(+)$ lactate. Determination with lactic dehydrogenase and DPN. In: Bergmeyer HU, editor. Methods of Enzymatic Analysis. New York: Academic Press, 1963: 266-70.

10. Yano K, Maruyama T, Makino N, Matsui H, Yanaga T. Effects of amiloride on the mechanical, electrical and biochemical aspects of ischemia-reperfusion injury. Mol Cell Biochem 1993; 121: 75-83.

11. Mitani S, Hashimoto H, Matsui H, Tokj Y, Okumura K, Ito T. Insulin as a determinant of cardiac but not skeletal muscle creatine kinase MB (abst). J Mol Cell Cardiol 1994; 26: CCXXIV.

12. Bernauer W. Digoxin induced release of creatine kinase from isolated guinea-pig hearts. NaunynSchmiedeberg's Arch 1979; 309: 83-8.

13. Sammel NL, Norris RM, Hughes CF, Johnson RN, Ashton NG, Elliott RL. Severity of canine myocardial infarcts in relation to indices of oxygen demand: preservation of myocardial creatine kinase activity by vagal stimulation and propranolol. Cardiovasc Res 1983; 17:50-60.

14. Rona G, Chappel CI, Balazs T, Gaudry R. An infarct-like myocardial lesion and other toxic manifestations produced by isoproterenol in the rat. Arch Pathol 1959; 67: 443-55.

15. Rona G, Chappel CI, Kahn DS. The significance of factors modifying the development of isoproterenol-induced myocardial necrosis. Am Heart J 1963; 66: 389-95.

16. Fleckenstein A, Janke J, Do'ring HJ, Pachinger O. Ca overload as the determinant factor in the production of catecholamine-induced myocardial lesions. In: Bajusz E, Rona G, editors. Cardiomyopathies. Baltimore: University Park Press, 1973: 455-66.

17. Benjamin IJ, Jalil JE, Tan LB, Cho K, Weber KT, Clark WA. Isoproterenol-induced myocardial fibrosis in relation to myocyte necrosis. Circ Res 1989; 65: 657-70.

18. Hakamata $\mathrm{N}$. Hamada $\mathrm{H}$, Ohsuzu $\mathrm{F}$, Nakamura $\mathrm{H}$. Cardiac-adrenergic signaling pathway alteration in isoproterenol-induced cardiac hypertrophy in male Sprague-Dawley rats. Jpn Heart J 1997; 38: 84957.

19. Mak IT, Kramer JH, Freedman AM, Tse SYH, Weglicki WB. Oxygen radical-mediated injury of myocytes-protection by propranolol. J Mol Cell Cardiol 1990; 22: 687-95.

20. Hermans AN, Glitsch HG, Verdonck $F$. The antagonistic effect of $\mathrm{K}^{+}$ond dihydro-ouabain on the $\mathrm{Na}^{+}$pump current of single rat and guinea-pig cardiac cells. J Physiol (Lond) 1995; 484: 617-28.

21. Shattock MJ, Bers DM. Rat vs. rabbit ventricle: Ca flux and intracellular $\mathrm{Na}$ assessed by ion-selective microelectrodes. Am J Physiol 1989; 256: C813-C822.

22. Liu X, Engelman RM, Agrawal HR, Das DK. Preservation of membrane phospholipids by propranolol, pindolol, and metoprolol: a novel mechanism of action of beta-blockers. J Mol Cell Cardiol 1991; 23: 1091-100.

23. Fujioka $H$, Yoshihara $S$, Tanaka $T$, et al. Enhancement of post-hypoxic contractile and metabolic recovery of perfused rat hearts by $d l$-propranorol: possible involvement of non-beta-receptor mediated activity. J Mol Cell Cardiol 1991; 23: 949-62.

24. Okumura K, Ogawa K, Satake T. Pretreatment with chlorpromazine prevents phospholipid degradation and creatine kinase depletion in isoproterenol-induced myocardial damage in rats. $\mathrm{J}$ Cardiovasc Pharmacol 1983; 5: 983-8. 